\title{
The FIRST Unbiased Survey for Radio Stars
}

\author{
David J. Helfand and Scott Schnee \\ Department of Astronomy, Columbia University, New York, NY 10027 \\ Robert H. Becker \\ Department of Physics, University of California, Davis \\ and \\ Institute for Geophysics and Planetary Physics, LLNL \\ Richard L. White \\ Space Telescope Science Institute, Baltimore, MD 21218 \\ Richard G. McMahon \\ Institute of Astronomy, University of Cambridge, Cambridge CB3 OHA, UK
}

\begin{abstract}
Comparison of the VLA FIRST survey with various catalogs of bright stars allows an examination of the prevalence of stellar radio emission independent of optical selection criteria. This FIRST unbiased survey for radio stars covers nearly $5000 \mathrm{deg}^{2}$ of the northern sky to a flux density limit of $0.7 \mathrm{mJy}$ at $20 \mathrm{~cm}$. Using astrometric catalogs which include proper motion information, we have detected 26 stellar radio sources, doubling the number of such objects previously known in this region of high-latitude sky. We also show that, in the absence of good proper motions, even the $1^{\prime \prime}$ precision of the FIRST positions is insufficient to avoid crippling chance coincidence rates. We calculate the fraction of radio detections as a function of stellar magnitude, and show that, when proper motions from the Guide Star Catalog-II become available, the number of stellar radio source detections should increase fourfold.
\end{abstract}

Subject headings: stars:activity — radio continuum — surveys 


\section{Introduction}

It has been 56 years since the Sun was recognized as the first radio-emitting star (Hey 1973). The subsequent history of stellar radio astronomy can be neatly divided into two eras of 28 years each (cf. Hjellming 1988). In the first (1942-1970), sensitivity limitations and source confusion left the Sun as the sole member of the class of radio stars, despite early attempts to categorize all radio sources with this epithet (Ryle, Smith, and Elsmore 1950) and, later, unconfirmed reports of enormous flares from nearby red dwarfs (Lovell 1964) ${ }^{1}$. The dawn of the second era ocurred in 1970 with the opening of the Westerbork and Green Bank radio interferometers which, for the first time, provided routine source localization to a few arcseconds and pushed sensitivity thresholds to $\sim 10 \mathrm{mJy}$. While still insufficient to detect a solar clone at even a few parsecs, observations with these instruments quickly identified several classes of stellar radio sources: novae (Hjellming and Wade 1970), Algol-type systems (Wade and Hjellming 1972), RSCVn binaries (Hjellming and Blankenship 1973), massive O-star binaries (Gibson and Hjellming 1974), and even some of the newly discovered Xray binary systems (Hjellming and Wade 1971; Braes and Miley 1971). With the advent of the Very Large Array (VLA) ten years later, stellar radio astronomy blossomed such that there are now nearly 1000 stars that have been detected at least once as a source of centimetric emission.

In virtually all cases, however, stellar radio detections have resulted from targeted observations of stars selected on the basis of their optical and/or X-ray properties. While this approach has expanded the number of stellar types detected to include pre-main sequence stars (Cohen, Bieging, and Schwartz 1982), ultra-fast rotating ZAMS G and $\mathrm{K}$ stars (Lim and White 1995), various types of cataclysmic variables (e.g.,Chanmugam and Dulk 1982; Padin, Davies, and Bode 1985; Turner 1985), chemically peculiar A and B stars (Drake et al. 1987), and numerous classes of objects displaying strong stellar winds (White and Becker 1982; Abbott et al. 1986), such samples suffer from two drawbacks: 1) the construction of stellar radio luminosity functions can be compromised by the

\footnotetext{
${ }^{1}$ dMe flare stars were, in fact, subsequently detected both in outburst and in quiescence (Spangler, Shawhan, and Rankin 1974 and references therein; Davis et al. 1978; Gary and Linksy 1981)
}

optical selection criteria, and 2) the discovery of new and unexpected types of stellar radio sources is, by definition, excluded. An unbiased, large-area sky survey avoids both these problems, and it is the first results from such a survey that we describe here.

It has taken over half a century to begin such a survey for the simple reason that most stellar radio sources are faint. Prior to the inauguration of the National Radio Astronomy Observatory's ${ }^{2}$ VLA sky survey program in 1993, the best available sky surveys were constructed from single-dish and meterwave interferometric instruments, and had sensitivities in the range 30-100 mJy. Fewer than twenty of the $\sim 100,000$ sources catalogued in these efforts are stars. The factor of fifty improvement offered by the Faint Images of the Radio Sky at Twenty-cm (FIRST) we are now collecting with the VLA offers us the opportunity to construct the FIRST unbiased stellar survey with a realistic chance of substantially enhancing our knowledge of the radio emission from stars.

Sensitivity alone, however, is not sufficient for a successful survey for radio stars; astrometric accuracy is also essential. The low surface density of optically bright stars might suggest that arcsecond radio positions are not important, but this is not the case: the rarity of stellar radio sources coupled with the high surface density of faint extragalactic radio sources conspire to drive the chance coincidence rate to an unacceptable level for radio positions worse than $\sim 1^{\prime \prime}$ (cf. Condon, Kaplan, and Yin 1997), even, as we show below, when the optical magnitude limit of the stellar sample is relatively bright $\left(m_{v} \sim 10\right)$. Thus, it is our radio survey's unique combination of high sensitivity and high astrometric accuracy that allows us to carry out this FIRST unbiased survey for radio stars.

In section 2, we describe briefly the FIRST survey, concentrating on its features germane to a stellar radio source search. We then proceed to describe in turn the optical catalogs we have used to identify radio emitting objects, including a discussion of how matches were selected for each and the resulting list of identifications. We detect a total of 26 stellar radio sources in our $4760 \mathrm{deg}^{2}$ area, doubling the number of objects known heretofore in this high Galactic lat-

\footnotetext{
${ }^{2}$ The National Radio Astronomy Observatory is a facility of the National Science Foundation operated under cooperative agreement by Associated Universities, Inc.
} 
itude region. Section 4 relates how, using stars with high-precision positions, we have searched the FIRST image database in order to lower the survey threshold to $0.7 \mathrm{mJy}$. The effect of radio star variability on our search is explored in section 5. Following a brief discussion of the radio stars we have detected, we highlight in section 6 the prospects for a substantial expansion in the number of FIRST stellar identifications which will derive from a comparison with the Guide Star Catalog-II. We conclude ( $\$ 7$ ) with a summary of our survey.

\section{The FIRST Survey}

Since April of 1993, we have been using the VLA to construct the centimetric equivalent of the Palomar Observatory Sky survey. Observations are conducted in the B configuration in bandwidth-synthesis mode with fourteen $3 \mathrm{MHz}$ channels centered at $1400 \mathrm{MHz}$ $(20 \mathrm{~cm})$. Overlapping snapshots of $165 \mathrm{~s}$ duration are conducted along a strip of constant declination each day to produce a raw database of "grid" images, one for each pointing. Subsequent processing produces a set of $35^{\prime} \times 45^{\prime}$ "coadded" images from the thirteen overlapping fields which contribute to each location; the catalog of radio sources derived from these images forms the basis of this survey for radio stars.

To date, we have reduced and analyzed data from a region covering $\sim 4150 \mathrm{deg}^{2}$ in the north Galactic cap above Galactic latitude $25^{\circ}$ between declinations $+22^{\circ}$ and $+58^{\circ}$, as well as two narrow strips in the southern Galactic cap centered near declinations $0^{\circ}$ and $-9^{\circ}$ covering a total of $610 \mathrm{deg}^{2}$. The $5.4^{\prime \prime}$ synthesized beam ${ }^{3}$ of the array in this mode allows us to detect source structure down to scales of $2.5^{\prime \prime}$, and, along with our careful treatment of astrometric calibration, allows us to obtain positional accuracies for all sources down to the survey threshold at $1 \mathrm{mJy}$ of better than $1^{\prime \prime}$ (90\% confidence). As we show below, this positional accuracy is crucial in reducing the rate of false coincidences with optical stars to a manageable level.

A complete description of the survey observations, the data reduction, and the procedures used in constructing the source catalog may be found in Becker,

\footnotetext{
${ }^{3}$ In the northern portion of the survey, a circular 5.4" convolving beam is used to construct the CLEANed images; in the southern Galactic cap, the restoring beam is $5.4^{\prime \prime} \times 6.4^{\prime \prime}$ to take into account the squint of the Array's synthesized beam at southern declinations.
}

White, and Helfand (1995; hereafter BWH) and White et al. 1997 (hereafter WBHG). Public access to all images and catalogs, along with the survey coverage map and other relevant details, are available through the FIRST Website at http://sundog.stsci.edu/.

\section{Matching with Selected Stellar Catalogs}

Over $99.9 \%$ of the 437,429 sources in the current FIRST catalog are extragalactic objects. Our principal means of obtaining optical identifications for these sources is by constructing a match based on positional coincidence with the Cambridge Automated Plate Machine (APM) catalog of optical objects derived from the POSS I plates (McMahon et al. 1998). For stellar radio sources, however, this procedure fails owing to the significant proper motions (typically several arcseconds) experienced by nearby stars in the forty years between the two surveys (see $\S 3.5$ below). Thus, we restrict our final list of identifications in this paper to stars found in optical catalogs which contain proper motion information.

\subsection{Hipparcos}

The June 1997 release of the Hipparcos catalog contains positions, proper motions, and parallax measurements for $\sim 120,000$ selected stars brighter than $m_{v} \sim 11$ (Perryman et al. 1997). For each of the 13,302 Hipparcos stars lying within the FIRST survey area, we have calculated stellar positions for the epoch of the relevant FIRST observation and then matched the resulting catalog to the FIRST radio catalog (98Feb04 version).

It should be noted that the FIRST observation epoch is usually, but not always, unambiguous; each coadded image from which the radio catalog is derived $(\mathrm{BWH})$ is, as noted above, a weighted mean of thirteen overlapping pointings and, while typically all such pointings are made within a week or two of each other, at the boundaries of each year's coverage and in the case of the small number of fields reobserved in later years when the original data were corrupted, a single coadded image can contain data taken up to three years apart. In the vast majority of cases, the net proper motion over even this maximum interval is a small fraction of the synthesized beam, and the smearing of the source which results is undetectable. Furthermore, only one star with a proper motion $>0.7^{\prime \prime}$ per year (leading to a potential centroid shift of $>1^{\prime \prime}$ in noncontemporaneous images) 
fell within $30^{\prime \prime}$ of a FIRST source (and this source is an obvious extragalactic triple), rendering this issue largely academic. As the FIRST survey continues, however, the interval between the Hipparcos and FIRST observation epochs will grow. Thus, in calculating FIRST source epochs, we have derived a fluxweighted value which takes into account the facts that 1) for a variable source, the catalog position may be dominated by a single observation in which the source is bright, and 2) for all sources, the dominant contribution to the cataloged properties comes from the observation(s) in which the source is closest to the grid pointing field center (see Table 1, footnote 3 ).

We have calculated the positional uncertainties for the stars by adding in quadrature the quoted uncertainties in the optical coordinates and in the star's proper motion after extrapolating to the FIRST epoch from 1991.25 (the Hipparcos standard epoch). Position uncertainties for potentially matching radio sources depend only on their extent and the signalto-noise ratio at which they were detected, and were computed as described in WBHG. All stars whose offsets from the nearest catalogued radio source were $<35^{\prime \prime}$ were tabulated in order to derive an empirical false coincidence rate; 304 objects met this criterion. Sources for which the offset was less than three times the quadrature sum of the stellar and radio source positional uncertainties were retained as potential identifications.

Ten Hipparcos stars are coincident with a source in the FIRST catalog to better than $1.0^{\prime \prime}$; in all cases the match is good to within $2 \sigma$ of the combined uncertainties in the radio and optical positions. The expected false rate, derived from the matches between $2^{\prime \prime}$ and $35^{\prime \prime}$, as well as from a match to a fake catalog of radio sources offset by $5^{\prime}$ from the real catalog, is $0.24 \pm 0.01$ matches. Three additional stars lie between $1^{\prime \prime}$ and $2^{\prime \prime}$ from FIRST catalog entries and have positions consistent within $2.2 \sigma$. The expected rate for chance coincidences at separations $<2^{\prime \prime}$ is $0.96 \pm 0.04$, so it is possible that one of these is a false match. However, an a posteriori calculation of the false rate using the magnitude distribution of the detected stars suggests this is unlikely: all 13 stars have $m_{v}<8.5$, and the false rate within $2^{\prime \prime}$ for this subset of the Hipparcos catalog is only 0.40 .

Two additional stars with offsets of $4.9^{\prime \prime}$ and $7.8^{\prime \prime}$, respectively, have positions consistent to between 2 and $3 \sigma$ (the uncertainties are large because of large proper motions); however, since the total number of stars between $2^{\prime \prime}$ and $10^{\prime \prime}$ is 24 with an expected false rate of 23.1 , it is not statistically likely that these are real detections.

Of the thirteen matches, all are radio point sources ${ }^{4}$ with flux densities $<6 \mathrm{mJy}$, lending further credence to the identifications; indeed, if we restrict the match to radio sources with major axes $<2.5^{\prime \prime}$ (our limit for detecting source extent), the false rates are reduced by an additional factor of 2.2 to 0.18 within $2^{\prime \prime}$. (In contrast, the two stars with larger but consistent offsets mentioned above are both extended.) Thus, we are reasonably confident that all thirteen stars are truly radio emitters. Six of these objects have been detected previously at radio wavelengths and seven are newly discovered radio stars.

Given the well-established correlation between stellar X-ray and radio luminosities (e.g., Gudel and Benz 1993), the detection of our candidate radio stars as $\mathrm{X}$-ray sources would add yet another piece of confirming evidence that the radio detections are correct. We have thus queried the High Energy Astrophysics Science Archive Research Center's Master Catalog of X-ray sources at the positions of each of our detected stars. Eleven of the thirteen are detected in the ROSAT All-Sky Survey Bright Source Catalog (RASS-BSC Voges et al. 1996); several of these were also observed by previous X-ray astronomy missions. In contrast, only $1.4 \%$ of all Hipparcos stars in the FIRST region are detected in the RASS-BSC.

Of the two stars not previously reported as Xray sources, one is a known binary and IRAS source (FIRST J141600.0+564120), and the limit on its radio to X-ray flux ratio is not far outside the range observed for the stellar radio source population as a whole. The other star, however, is somewhat extreme (FIRST J142104.8+385914). It is the brightest radio coincidence we detect and, while four independent observations over six days show it to be variable at the $20 \%$ level (see §5), we have clearly not simply recorded a single, anomalously bright radio flare. In the $L_{x}-L_{r}$ diagram (Gudel and Benz 1993), this star lies a factor of 30 below the mean X-ray luminosity for its radio brightness, and nearly an order of magnitude below the most extreme example of a radio-bright/X-rayquiet star. Subsequent observations may reveal that

\footnotetext{
${ }^{4}$ The star at $\mathrm{RA}(\mathrm{J} 2000)=14^{h} 15^{m} 59^{s} .89, \quad \operatorname{Dec}(\mathrm{J} 2000)=$ $+56^{\circ} 41^{\prime} 21^{\prime \prime} .7$ is listed in the FIRST catalog as having an extent of $8^{\prime \prime}$; however, examination of the FIRST images shows a point-like source superposed on a low-level stripe in the map, which is the cause of the spurious major axis reported.
} 
this is an example of a new type of radio star which optically selected samples have missed ${ }^{5}$.

The thirteen radio star detections are listed in Table 1 , where we include the radio source position, its epoch, its offset from the Hipparcos position, and the $1 \sigma$ uncertainty in the offset which, in this case, is dominated by the radio source positional uncertainty. Also listed are the star's name (if any), magnitude and parallax distance from the Hipparcos catalog, the stellar system's classification (if known), and the star's spectral type. The $20-\mathrm{cm}$ peak flux density of the source from the FIRST catalog is given in column 9; the same quantity from the NRAO VLA Sky Survey (Condon et al. 1998) is also given for the few sources detected, along with an indication if the star is a previously known radio source. The star's X-ray count rate in the RASS-BSC is also included. The provenance of the star (in this case, the Hipparcos catalog) and the radio source (in this case, the FIRST cata$\log$ ) are given in columns 12 and 13. Optical and radio variability information is supplied in the final column. A more detailed description of all this information is provided in the footnotes to the Table.

\subsection{Tycho}

The Tycho catalog, derived from a separate experiment on the Hipparcos mission, contains 1,058,332 objects down to $m_{v} \sim 13$ (Høg et al. 1997). Identical procedures to those adopted for the Hipparcos catalog were used to compare this catalog with FIRST radio sources. Of the 74,889 Tycho stars which lie in the FIRST survey region, 1730 fell within $35^{\prime \prime}$ of a FIRST source; no high proper motion Tycho stars were within this radius.

In addition to the thirteen Hipparcos detections within $2^{\prime \prime}$, ten additional entries in the Tycho catalog have offsets of $\leq 2.0^{\prime \prime}$ from FIRST sources; all match to within their respective $3 \sigma$ uncertainties. The expected false rate, derived as above, is 5.7, suggesting some of these matches could be real. However, the match to the $11^{\text {th }}$ magnitude "star" at RA $(\mathrm{J} 2000)=$ $16^{h} 44^{m} 29^{s} .49$, Dec (J2000) $=23^{\circ} 48^{\prime} 00^{\prime \prime} .1$ is an extended source with an integrated flux density $S_{20 \mathrm{~cm}}=$ $300 \mathrm{mJy}$; in fact, this radio source is the planetary nebula NGC 6210 (as is the Tycho catalog entry). A second source, FIRST 020920.8-100759, matches

\footnotetext{
5 or that it harbors little green persons broadcasting their presence.
}

the galaxy NGC 833. (Although $\mathrm{SIMBAD}^{6}$ lists a double star less than $2^{\prime \prime}$ away - presumably the Tycho target - no such object appears on the Digitized Sky Survey image; the galaxy is a plausible identification for the radio source.) While these are true radio detections of Tycho catalog objects, they are clearly not radio stars. Thus, with 8 remaining detections and an expected false rate of $\sim 6$, at most a few are real matches. Examination of the radio images shows that two are classical extragalactic radio doubles, and a third is an unflagged sidelobe; these account for half of the expected number of chance coincidences. The other six matches are all consistent with point sources, are isolated, and have flux densities $\leq 2 \mathrm{mJy}$. None has previously been reported as a radio emitter, although one is detected in the RASS-BSC, strongly suggesting it is a real detection (only $0.4 \%$ of all Tycho stars in our region are BSC sources); we include this object in Table 1. In view of the fact that several of the remaining stars may be chance coincidences, we segregate these from the far more secure Hipparcos detections in Table 2A; Table $2 \mathrm{~B}$ is reserved for the clearly non-stellar matches from the Tycho catalog which we record for completeness.

\subsection{PPM}

Prior to the recent release of the Hipparcos data, the Positions and Proper Motions Catalog (Röser and Bastian 1991) was the primary reference for deriving current epoch positions of stars brighter than $m_{v} \sim 12$. The errors on the positions and proper motions are in general considerably larger than those now available, but we included a match as a test of the completeness of the two newer catalogs. At a matching radius of $2^{\prime \prime}$, all of the PPM candidate radio sources appear in the Hipparcos list and, as a result, we will not discuss further data from this catalog.

\subsection{Stars within $25 \mathrm{pc}$}

Gliese and Jahreiss 1991 have compiled from the literature a list of all stars whose parallax measurements place them at distances of less than $25 \mathrm{pc}$ from the Sun. The catalog by no means represents a complete volume-limited sample, but it does include

\footnotetext{
${ }^{6}$ the Set of Identifications, Measurements and Bibliography for Astronomical Data system is operated by the Centre de Données Astronomiques under agreement between the French Institut National des Sciences de l'Univers and Université Louis Pasteur, Starsbourg.
} 
TABLE 1

Stellar Radio Sources Found in the FIRST Survey

\begin{tabular}{|c|c|c|c|c|c|c|c|c|c|c|c|c|c|c|c|c|c|}
\hline \multirow{3}{*}{$\begin{array}{c}\mathrm{RA}(2000) \\
\text { (1) }\end{array}$} & \multirow{3}{*}{$\begin{array}{c}\mathrm{DEC}(2000) \\
\text { (2) }\end{array}$} & \multirow{3}{*}{$\begin{array}{l}\text { Epoch } \\
\text { (199x) } \\
(3)\end{array}$} & \multirow{3}{*}{$\begin{array}{l}\text { Name } \\
\text { (4) }\end{array}$} & \multirow{3}{*}{$\begin{array}{l}\text { Class } \\
\text { (5) }\end{array}$} & \multirow{3}{*}{$\begin{array}{l}\text { Sp. Type } \\
\text { (6) }\end{array}$} & \multirow{3}{*}{$\begin{array}{l}\text { Mag } \\
\text { (V) } \\
(7)\end{array}$} & \multirow{3}{*}{$\begin{array}{l}\text { X-ray } \\
\text { (cps) } \\
\text { (8) }\end{array}$} & \multirow{3}{*}{$\begin{array}{l}\text { FIRST } \\
\text { (9) }\end{array}$} & \multirow{2}{*}{$\begin{array}{l}\text { Peak Flux (m) y) } \\
\text { NVSS }\end{array}$} & \multirow{3}{*}{$\begin{array}{l}\text { Other } \\
\text { (11) }\end{array}$} & \multicolumn{2}{|c|}{ Provenance } & \multirow{3}{*}{$\begin{array}{l}\text { offset } \\
\left({ }^{\prime \prime}\right) \\
(14)\end{array}$} & \multirow{3}{*}{$\begin{array}{l}\text { error } \\
\left({ }^{\prime \prime}\right) \\
(15)\end{array}$} & \multirow{3}{*}{$\begin{array}{l}\text { Dist } \\
\text { (pc) } \\
(16)\end{array}$} & \multicolumn{2}{|c|}{ Variability } \\
\hline & & & & & & & & & & & Optical & Radio & & & & Hipparcos & FIRST \\
\hline & & & & & & & & & (10) & & (12) & (13) & & & & (17) & (18) \\
\hline 011636.298 & -023000.90 & 5.35 & AY Cet & RSCVn & G5IIIe & 5.42 & 3.94 & 2.55 & $\cdots$ & $<0.2-5.2(6)$ & $\mathrm{Hs}$ & catalog & 0.39 & 0.73 & $78+4$ & binary & \\
\hline $\begin{array}{l}012250.315 \\
072133845\end{array}$ & $\begin{array}{l}004244.47 \\
260934.04\end{array}$ & $\begin{array}{l}5.29 \\
5.32\end{array}$ & $\begin{array}{l}\text { BI C et } \\
\text { V } 340 \mathrm{G} \mathrm{em}\end{array}$ & RSCVn & $\begin{array}{l}G 0 \\
G 2 V\end{array}$ & $\begin{array}{l}8.26 \\
7.53\end{array}$ & $\begin{array}{l}1.52 \\
0.28\end{array}$ & 2.17 & & $2.7-3.2(6)$ & $\mathrm{Hs}$ & catalog & 0.11 & 0.81 & $66+4$ & $\mathrm{u}$ & \\
\hline $\begin{array}{l}072133.845 \\
073435.879\end{array}$ & $\begin{array}{l}260934.04 \\
315318.88\end{array}$ & $\begin{array}{l}5.32 \\
6.32\end{array}$ & $\begin{array}{l}\sqrt{340} \mathrm{G} \mathrm{em} \\
\text { Castor AB }\end{array}$ & & $\begin{array}{l}\mathrm{G} 2 \mathrm{~V} \\
\mathrm{~A} 2 \mathrm{Vm}\end{array}$ & $\begin{array}{l}7.53 \\
1.58\end{array}$ & $\begin{array}{l}0.28 \\
3.70\end{array}$ & $\begin{array}{l}1.47 \\
0.76\end{array}$ & 6.5 & $0.4-0.6$ & $\begin{array}{l}\mathrm{HS} \\
\mathrm{Hs}\end{array}$ & $\begin{array}{l}\text { grid } \\
\text { image }\end{array}$ & $\begin{array}{l}0.86 \\
1.16\end{array}$ & $\begin{array}{l}0.29 \\
3.34\end{array}$ & $\begin{array}{c}232+67 \\
16+1\end{array}$ & $\begin{array}{l}\text { u } \\
\text { double }\end{array}$ & $<1.0-9.9$ \\
\hline 074318.648 & 285302.22 & 3.12 & Sig Gem & RSCVn & K 11111 & 4.23 & 8.08 & 1.99 & $\cdots$ & $<3.0-45(13)$ & $\mathrm{Hs}$ & catalog & 1.01 & 0.62 & $37+1$ & 4 & $1.9-3.6$ \\
\hline $\begin{array}{l}080235.793 \\
08\end{array}$ & 571626.26 & 7.14 & $54 \mathrm{Cam}$ & $R S C V n$ & $F 8 V$ & 6.48 & 1.35 & 1.02 & $x$ & $<0.2-5.0(6)$ & $\mathrm{Hs}$ & image & 1.02 & 1.57 & $102+8$ & micro & \\
\hline 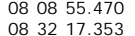 & $\begin{array}{l}324906.03 \\
291909.63\end{array}$ & $\begin{array}{l}4.18 \\
3.10\end{array}$ & $\begin{array}{l}\text { GJ } 1108 \text { B } \\
\text { SAO } 80232\end{array}$ & & $\begin{array}{l}\text { SM 3ve } \\
\text { G5 }\end{array}$ & $\begin{array}{l}11.4 \\
7.35\end{array}$ & $\begin{array}{l}0.78 \\
0.82\end{array}$ & $\begin{array}{l}2.47 \\
5.39\end{array}$ & & & $\begin{array}{c}25 p c \\
H s\end{array}$ & $\begin{array}{l}\text { catalog } \\
\text { catalog }\end{array}$ & $\begin{array}{l}1.27 \\
0.04\end{array}$ & $\begin{array}{l}0.53 \\
0.41\end{array}$ & $\begin{array}{c}21+1 \\
182+30\end{array}$ & NA & $\begin{array}{l}<1.0-5.9 \\
27-5.5\end{array}$ \\
\hline $\begin{array}{l}083217.353 \\
090327.030\end{array}$ & $\begin{array}{l}291909.63 \\
375029.51\end{array}$ & $\begin{array}{l}3.10 \\
4.21\end{array}$ & $\begin{array}{l}\text { SAO } 80232 \\
\text { SAO } 61224\end{array}$ & & $\begin{array}{l}\text { G5 } \\
\text { G0 }\end{array}$ & $\begin{array}{l}7.35 \\
7.04\end{array}$ & $\begin{array}{l}0.82 \\
0.66\end{array}$ & $\begin{array}{l}5.39 \\
1.67\end{array}$ & $\cdots$ & & $\begin{array}{l}\mathrm{Hs} \\
\mathrm{Hs}\end{array}$ & $\begin{array}{l}\text { catalog } \\
\text { catalog }\end{array}$ & $\begin{array}{l}0.04 \\
1.61\end{array}$ & $\begin{array}{l}0.41 \\
0.72\end{array}$ & $\begin{array}{c}182+30 \\
30+1\end{array}$ & $\ldots$ & $2.7-6.5$ \\
\hline 094112.056 & 483609.30 & 7.10 & & & & 10.88 & 0.25 & 1.69 & & & Ty & image & 0.63 & 0.57 & & & $<1.0-1.8$ \\
\hline $1000 \quad 01.736$ & 243309.61 & 5.34 & DH Leo & ByDra & Kov & 7.86 & 2.05 & 1.08 & $\ldots$ & $2.2(13)$ & Hs & & 0.85 & & $32+1$ & binary & \\
\hline 125601.653 & 381905.43 & 4.22 & $\alpha^{2} \mathrm{CVn}$ & & A 0spe & 2.89 & 0.08 & 0.85 & $\ldots$ & $<0.2(6)$ & $\mathrm{Hs}$ & image & 1.68 & 2.80 & $34+1$ & binary & \\
\hline 131037.001 & 355606.93 & 4.19 & RS CVn & RSCVn & $F 4 V$ & 8.07 & 0.89 & 0.80 & $\ldots$ & 1.8 & $\mathrm{Hs}$ & image & 1.70 & 2.55 & $108+12$ & periodic & \\
\hline 132132.297 & 385249.31 & 4.22 & BM CVn & RSCVn & G81II & 7.31 & 1.32 & 1.06 & $\cdots$ & E 05 & $\mathrm{Hs}$ & catalog & 0.35 & 1.00 & $111+9$ & u & \\
\hline $\begin{array}{l}1333046.831 \\
133146.726\end{array}$ & $\begin{array}{l}241358.42 \\
291637.77\end{array}$ & $\begin{array}{l}5.34 \\
3.12\end{array}$ & $\begin{array}{l}\text { FK Comae } \\
\text { G } 165-8\end{array}$ & & G21II & 8.15 & 0.18 & $\begin{array}{l}1.75 \\
2.51\end{array}$ & $\cdots$ & $<0.6-1.6$ & $\begin{array}{l}\mathrm{Hs} \\
25 \mathrm{c}\end{array}$ & $\begin{array}{l}\text { catalog } \\
\text { catalog }\end{array}$ & 0.63 & 0.63 & $234+55$ & binary & \\
\hline $\begin{array}{l}1333466.726 \\
133447.777\end{array}$ & $\begin{array}{l}291637.77 \\
371056.69\end{array}$ & $\begin{array}{l}3.12 \\
4.20\end{array}$ & $\begin{array}{l}\text { G 1 l65-8 } \\
\text { BH CVn }\end{array}$ & RSCVn & $\begin{array}{l}\text { dM } 4 \mathrm{e} \\
\text { F } 1 \mathrm{~V}\end{array}$ & $\begin{array}{l}14.1 \\
4.91\end{array}$ & $\begin{array}{l}0.41 \\
2.77\end{array}$ & $\begin{array}{l}2.51 \\
4.02\end{array}$ & 9.0 & $5.5-25.0$ & $\begin{array}{c}25 \mathrm{pc} \\
\mathrm{Hs}\end{array}$ & $\begin{array}{l}\text { catalog } \\
\text { catalog }\end{array}$ & $\begin{array}{l}0.26 \\
0.12\end{array}$ & $\begin{array}{l}0.26 \\
0.50\end{array}$ & $\begin{array}{l}<25 \\
45+1\end{array}$ & NA & \\
\hline $14 \quad 1600.035$ & 564120.86 & $\begin{array}{l}7.20 \\
7.14\end{array}$ & Dח CVח & ksevin & F8 & $\begin{array}{l}4.91 \\
6.68\end{array}$ & $\ldots$ & $\begin{array}{l}4.02 \\
1.31\end{array}$ & 9.0 & $0.5-23.0$ & Hs & $\begin{array}{l}\text { catalog } \\
\text { catal }\end{array}$ & $\begin{array}{l}0.12 \\
1.23\end{array}$ & $\begin{array}{l}0.50 \\
1.80\end{array}$ & $48+1$ & double & \\
\hline $14 \quad 2104.829$ & $\begin{array}{lll}38 & 59 & 14.91\end{array}$ & 4.22 & & & F 5 & 7.92 & & 5.59 & 6.8 & & $\mathrm{Hs}$ & catalog & 0.27 & 0.44 & $101+9$ & (n) & $5.5-7.1$ \\
\hline 142643.288 & 315214.76 & 4.16 & & & & 10.27 & 0.40 & 1.07 & & & Ty & catalog & 1.98 & 0.69 & & & (1) \\
\hline 154357.912 & 222023.25 & 6.28 & & & F8 & 8.10 & 0.13 & 0.93 & & & Hs & image & 0.42 & 3.42 & $134+39$ & u & \\
\hline 155844.004 & 253411.05 & 5.33 & MS Ser & BYDra & $K 2 V$ & 8.19 & 0.64 & 2.26 & & & $\mathrm{Hs}$ & catalog & 0.34 & 0.65 & $88+7$ & binary & \\
\hline $\begin{array}{l}161440.979 \\
213740.196\end{array}$ & $\begin{array}{l}335131.61 \\
\end{array}$ & $\begin{array}{l}4.17 \\
5.28\end{array}$ & Sig CrB & RSCVn & $\begin{array}{l}\text { Gove } \\
\text { dMe }\end{array}$ & 5.23 & 9.40 & 5.52 & 4.7 & <1.-120. & Hs & catalog & 0.11 & 0.87 & $22+1$ & u & $<1.5-6.8$ \\
\hline 223633.793 & -003352.20 & 5.31 & & & Go & $\begin{array}{l}14.4 \\
8.34\end{array}$ & 0.20 & $\begin{array}{l}2.42 \\
0.89\end{array}$ & $\cdots$ & & Hs & $\begin{array}{l}\text { catagg } \\
\text { image }\end{array}$ & $\begin{array}{l}1.64 \\
0.80\end{array}$ & $\begin{array}{l}0.80 \\
2.82\end{array}$ & $112+14$ & u & \\
\hline 225852.922 & -00 1857.53 & 5.31 & AZ Psc & & KO & 7.42 & 0.20 & 1.28 & & & $\mathrm{Hs}$ & catalog & 0.20 & 1.42 & $147+19$ & u & \\
\hline
\end{tabular}

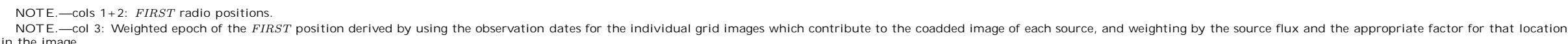
NOTE. - COI 4 (

NOTE.-col 4: Name, if any, of the star/ star system.

NOTE.- col 5: Classification, if known.

NOTE. - col 6: Spectral type as recorded in the SIMBAD database

NOTE. - col 8: Count rate recorded in the ROSAT All-Sky Survey Bright Source Catalog; adopting a spectral form including a Raymond-Smith thermal plasma with $T=1 \mathrm{keV}$ and neglible interstellar absorption, $1 \mathrm{cps} \sim 8 \times 10^{-12}$ erg $\mathrm{cm}^{-2} \mathrm{~s}^{-1}$.

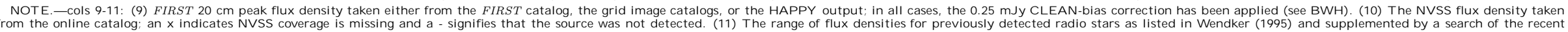
terature. The observation wavelength is noted in parentheses if it differs from $20 \mathrm{~cm}$.

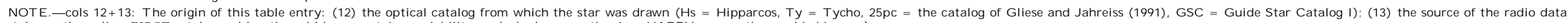
(catalog = the online FIRST catalog, grid = the grid image catalog variability analysis, image $=$ the deep HAPPY run on the coadded images).

NOTE. - cols 14+15: The offset between the radio and optical positions and the $1 \sigma$ error in the offset (see text).

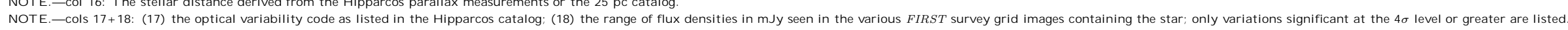


TABLE 2

UnConfirmed Radio Star Candidates

\begin{tabular}{|c|c|c|c|c|c|c|c|c|}
\hline \multirow[b]{2}{*}{$\mathrm{RA}(2000)$} & \multirow[b]{2}{*}{$\operatorname{Dec}(2000)$} & \multirow{3}{*}{$\begin{array}{c}\text { offset } \\
\left({ }^{\prime \prime}\right) \\
(3)\end{array}$} & \multirow{3}{*}{$\begin{array}{c}\text { error } \\
\left({ }^{\prime \prime}\right) \\
(4)\end{array}$} & \multirow{3}{*}{$\begin{array}{l}\text { Mag } \\
(5)\end{array}$} & \multirow{3}{*}{$\begin{array}{l}\text { Peak Flux } \\
\quad(\mathrm{mJy}) \\
(6)\end{array}$} & \multicolumn{2}{|c|}{ Provenance } & \\
\hline & & & & & & Optical & Radio & \\
\hline (1) & $(2)$ & & & & & (7) & $(8)$ & \\
\hline \multicolumn{9}{|c|}{ A. Unrefuted Candidate Star Detections } \\
\hline 085242.68 & +350101.5 & 1.67 & 0.32 & 10.2 & 2.1 & Ty & catalog & \\
\hline 094953.49 & +413247.4 & 1.75 & 1.72 & 9.5 & 0.9 & $\mathrm{Hs}$ & image & \\
\hline 102056.13 & +294143.2 & 1.44 & 0.46 & 10.8 & 1.3 & Ty & catalog & \\
\hline 121915.88 & +250335.1 & 1.98 & 2.63 & 7.8 & 0.9 & Hs & image & \\
\hline 124600.40 & +494916.8 & 1.96 & 0.59 & 9.9 & 1.2 & Ty & catalog & \\
\hline 150044.32 & +371348.3 & 1.91 & 0.40 & 11.2 & 1.7 & Ty & catalog & \\
\hline 163742.76 & +232423.6 & 1.32 & 0.35 & 10.6 & 4.1 & Ty & grid & \\
\hline & & & & & & & & \\
\hline 020920.86 & -100759.7 & 1.01 & 0.45 & 11.4 & 1.7 & Ту & catalog & galaxy (NGC 833) \\
\hline 020942.85 & -101102.5 & 1.55 & 0.11 & 11.4 & 28.5 & Тy & grid & galaxy (NGC 839) \\
\hline 071913.27 & +573552.4 & 1.80 & 0.95 & 11.3 & 1.1 & Ty & grid & noise \\
\hline 081609.47 & +481028.1 & 1.47 & 1.52 & 10.6 & 8.4 & Ty & grid & probable sidelobe \\
\hline 083050.67 & +241315.6 & 1.99 & 0.72 & 10.6 & 3.0 & Ty & grid & probable sidelobe \\
\hline 083307.95 & +552127.6 & 1.02 & 0.78 & 9.5 & 4.0 & $\mathrm{Hs}$ & grid & noise \\
\hline 084600.03 & +540303.8 & 1.76 & 2.71 & 10.9 & 10.3 & Ty & grid & noise \\
\hline 090905.32 & +374946.7 & 1.27 & 0.92 & 10.6 & 2.4 & Ty & grid & probable sidelobe \\
\hline 092639.88 & +385438.5 & 0.72 & 1.03 & 9.5 & 8.9 & Hs & grid & probable sidelobe \\
\hline 093253.26 & +353744.2 & 1.90 & 0.22 & 10.5 & 3.0 & Ty & catalog & extragalactic double \\
\hline 112940.05 & +501205.6 & 1.67 & 1.45 & 9.2 & 1.4 & Тy & grid & noise \\
\hline 124406.15 & +405025.6 & 1.44 & 0.91 & 10.4 & 1.8 & Ty & grid & probable sidelobe \\
\hline 132836.99 & +315755.8 & 1.89 & 0.89 & 10.2 & 1.5 & Ty & grid & noise \\
\hline 142514.23 & +413729.9 & 1.24 & 0.72 & 10.4 & 1.2 & Ty & grid & probable sidelobe \\
\hline 145611.95 & +52 1829.4 & 1.78 & 0.82 & 11.0 & 3.8 & Ty & grid & probable sidelobe \\
\hline 155500.45 & +452201.4 & 1.84 & 0.60 & 10.4 & 1.2 & Ty & catalog & ruled out by reobservation ${ }^{a}$ \\
\hline 163039.68 & +561015.3 & 0.99 & 0.59 & 10.3 & 1.3 & Ty & catalog & extragalactic double \\
\hline 164429.49 & +234800.1 & 0.84 & 0.10 & 10.4 & 101.3 & Тy & catalog & planetary nebula (NGC 6210) \\
\hline
\end{tabular}

a This radio source was observed at $3.6 \mathrm{~cm}$ by one of us (SS) and found to lie at least $0.65^{\prime \prime} \pm 0.1^{\prime \prime}$ from the stellar position even if the most favorable $3 \sigma$ errors in position and proper motion were adopted.

Note.- See footnotes to Table 1 for definitions of the parameters cited. 
TABLE 3

Previously Detected Radio Stars in the FiRSt Survey Region not Detected in FirSt

\begin{tabular}{|c|c|c|c|c|c|c|c|}
\hline $\begin{array}{c}\text { Star Name } \\
\text { (1) }\end{array}$ & $\begin{array}{c}\mathrm{RA}(2000) \\
(2)\end{array}$ & $\begin{array}{c}\operatorname{Dec}(2000) \\
(3)\end{array}$ & $\begin{array}{c}\text { Flux Range } \\
\text { (mJy) } \\
(4)\end{array}$ & $\begin{array}{l}\text { Obs. } \lambda \\
(\mathrm{cm}) \\
(5)\end{array}$ & $\begin{array}{l}\text { FIRST UL } \\
(\mathrm{mJy}) \\
(6)\end{array}$ & $\begin{array}{c}\text { Type } \\
(\mathrm{cps}) \\
(7)\end{array}$ & $\begin{array}{c}\text { X-Ray Flux } \\
\text { (8) }\end{array}$ \\
\hline HD7276 & 011301.55 & 010445.9 & $<7.5-17.3$ & 3.6 & 0.70 & Chromospherically active & \\
\hline Pollux & 074521.31 & 280136.8 & $<0.3-0.6$ & 6 & 0.67 & K0IIIb Pollux & 0.06 \\
\hline RU Cnc & 083730.20 & 233341.8 & $0.6-6.0$ & 6 & 0.64 & RS CVn & 0.11 \\
\hline WY Cnc & 090155.55 & 264124.0 & $0.4-0.5$ & 6 & 0.67 & Algol-type & 0.46 \\
\hline ST Lmi & 110539.33 & 250629.3 & $<0.2-2.0$ & 6 & 0.64 & Cataclysmic Variable & $0.20^{\mathrm{a}}$ \\
\hline UX Com & 130133.12 & 283754.5 & $<0.4-1.1$ & 6 & 0.55 & RS CVn & 0.20 \\
\hline ZZ Boo & 135609.88 & 255507.5 & $<0.5-9.0$ & 6 & 0.67 & RS CVn & $0.006^{\mathrm{b}}$ \\
\hline$\alpha \mathrm{CrB}$ & 153440.83 & 264257.1 & 0.6 & 6 & 0.67 & Algol-type & 0.12 \\
\hline $\mathrm{T} \mathrm{CrB}$ & 155930.20 & 255511.8 & $3.5-22.0$ & 13 & 0.70 & Symbiotic star & $\ldots$ \\
\hline HR6164 & 162838.41 & 415254.2 & 0.1 & 3.6 & 0.73 & Semiregular Variable & $\cdots$ \\
\hline V792 Her & 171025.58 & 485757.6 & $0.2-2.7$ & 6 & 0.64 & RS CVn & 0.37 \\
\hline Ross 868 & 171953.97 & 262949.2 & $<0.4-0.7$ & 20 & 0.70 & dMe flare star & 0.59 \\
\hline
\end{tabular}

${ }^{a}$ From the EXOSAT High Galactic Latitutde Survey.

${ }^{b}$ From a ROSAT PSPC pointed observation.

Note.-Cols 2-5: as in Table 1.

Note.-Col 6: $3 \sigma$ upper limit calculated from the relevant FIRST survey image.

Note.-Col 8: RASS-BSC count rate unless otherwise noted (see footnotes to Table 1 for conversion to cgs flux). 
proper motions for each entry, allowing us to calculate a FIRST-epoch position; since this list includes many stars fainter than the Tycho survey limit, we have included it in this study. A total of 571 stars from this tabulation fall within the confines of the FIRST survey, of which nearly half are not found in any of the above catalogs. Again, positional uncertainties were calculated by combining the position and proper motion uncertainties in quadrature. Since these uncertainties were often quite large (e.g., owing to early epoch proper motion measurements), we examined a contour plot of the radio image for a $1^{\prime}$ region around each star to assure that no potential candidates with flux densities $>0.7 \mathrm{mJy}$ ( ten stars for which there was a source within this region were followed up in order to ascertain the best astrometric parameters available from the literature.

This procedure yielded four matches with offsets of $<2^{\prime \prime}$, two of which are in Hipparcos and two of which are new. The expected false rate is only 0.03 matches within this radius, making the IDs virtually certain. While neither of the two new stars has been previously reported as a radio emitter, one is classified as a BY Draconis system and the other is a dMe star with strong $\mathrm{Ca} \mathrm{H} \& \mathrm{~K}$ emission. Both are radio point sources with peak flux densities of $\sim 2.5 \mathrm{mJy}$, and both are bright X-ray sources. Their other properties are summarized in Table 1 where their provenance is listed as "25pc sample". The remaining six stars all had final offsets between $10^{\prime \prime}$ and $30^{\prime \prime}$, consistent with the expected false rate, and are not considered further here.

\subsection{The Guide Star Catalog}

The Guide Star Catalog-I (GSC) is a compilation of all optical objects brighter than $m_{v} \sim 15$ derived from scans of the Quick-V Palomar $(\sim 1982)$ and UK SERC J $(\sim 1975$ and $\sim 1982)$ Survey plates (Lasker et al. 1990). In WBHG, we presented preliminary results for a match of the GSC to the first $1550 \mathrm{deg}^{2}$ of the FIRST survey. Nearly 500 objects classified as "nonstellar" (i.e., galaxies) were detected in FIRST with an expected chance coincidence rate of less than 1. In addition, 182 objects listed in the GSC as stellar also had FIRST counterparts, although we showed that the majority of these were misclassified galaxies, and that most of the remaining previously uncataloged objects were statistically indistinguishable in their radio and optical properties from the known galaxies. Only 12 objects were certifiably Galactic stars, only modestly in excess of the expected false rate. The potentially significant proper motions in the 10- to 20-year interval between the GSC plates and the FIRST survey required us to adopt a looser matching criterion of $3^{\prime \prime}$ for that analysis.

Any match between FIRST and the GSC is, then, problematic for two reasons: proper motions and misclassification of galaxies as stars. Since a substantial fraction of bright galaxies are detected by FIRST, even a small percentage of misclassifications in the GSC can lead to contamination which overwhelms the small number of real stellar radio source identifications. Nonetheless, with the available radio survey area now tripled in size, and the benefit of the Hipparcos data to provide unambiguous identifications, we have undertaken a reanalysis of the GSC-FIRST match. For each of the $\sim 2400$ GSC objects which matched a FIRST radio source to within $2^{\prime \prime}$, we produced $2^{\prime}$ - square cutouts from both the Digitized Sky Survey and the FIRST images. Each was examined by two of us (RHB and DJH) to extract a list of objects that were not obviously galaxies. A total of 204 objects remained after this initial screening. We performed searches on each position in the $\mathrm{NED}^{7}$ and SIMBAD databases in order to identify remaining extragalactic objects.

Of the 204 objects within $2^{\prime \prime}$ remaining after visual inspection, 15 were bona fide radio stars discovered in the catalog matches chronicled above. Another 64 can be confidently eliminated as galaxies with measured redshifts listed in NED (half classified as galaxies and half as stars in the GSC). An additional 34 objects are almost certainly galaxies - either they are listed in NED as such (albeit, without measured redshifts) or they are IRAS sources with the far-infrared colors typical of galaxies (more than two-thirds of this group are classified as stellar in the GSC). Finally, eight other objects are identified quasars (all appropriately classified as stellar). Thus, we are left with 82 objects, more than two-thirds of which are classified as stars.

In order to decrease further the extragalactic contamination, we checked the classifications of these objects in the APM catalog (see McMahon et al. 1998 for a discussion of the APM classification reliability). Twenty-two objects were classified as non-stellar on

\footnotetext{
${ }^{7}$ the NASA/IPAC Extragalactic Database is operated by the Jet Propulsion Laboratory, California Institute of Technology, under contract with the National Aeronautics and Space Administration.
} 
both the red and blue POSS-I plates; since only one of the 23 known stars or quasars discussed above was classified as non-stellar on both plates, it seems reasonable to eliminate these 22 from further consideration. Likewise, 28 of the sources are classified as stellar on both plates, and since only $1 / 98$ of the galaxies or probable galaxies discussed above were so classified, these objects are likely stellar candidates. This leaves 32 objects classified as stellar on one plate and non-stellar on the other for which no other information is available. Sixty-two percent of the known stars and quasars and $43 \%$ of the known or probable galaxies also fall in this category. For completeness, we retained these objects as candidates.

There remain, then, 60 candidate radio stars from the GSC match that are not eliminated by the above analysis. One of these stars is detected as a bright Xray source in the RASS-BSC. An optical spectrum obtained as part of a followup program of BSC-selected FIRST sources shows it to be a dMe star, and we include this as a definite detection in Table 1.

A false rate for the GSC was derived by matching with a false catalog of FIRST positions generated by shifting each entry $5^{\prime}$. As we did not repeat the elaborate filtering operation described above on this false match set, the rate we derive is a conservative upper limit. However, it is a reasonable estimate since the categories of sources the screening eliminated make up a minor portion of the GSC catalog: 1) the number of quasars in the GSC is tiny, 2) the number of galaxies in the whole catalog is also small (we find a large number in the real FIRST match because the radio survey preferentially selects galaxies), and 3) the number of nominal GSC stars classified as galaxies on both POSS plates by the APM is only $\sim 8 \%$. The expected number of false matches to objects classified as stellar in the GSC is $\sim 60$ within our adopted 2.0" radius, essentially equal to the number of new GSC stellar candidates. We do not list these here, because, while up to $\sim 10$ may be real detections, the chance coincidence rate is too high for any useful statements to be made.

\section{Image Database Search}

As described in WBHG, the FIRST source catalog has a flux density threshold of $1.0 \mathrm{mJy}$ and a source significance threshold of $5 \sigma$. Owing to the presence of "CLEAN bias" in VLA images (see BWH for a discussion), a 1.0 mJy threshold corresponds to a mea- sured map flux density of $0.75 \mathrm{mJy}$. The survey is $>95 \%$ complete for point sources at this threshold. However, the median image rms is $0.14 \mathrm{mJy}$, and a significant fraction of the survey area has noise levels of $\sim 0.10 \mathrm{mJy}$. In addition, given a precise optical position for a source of interest, lowering the source significance threshold below $5 \sigma$ is feasible, since the number of trials performed is drastically lower than in an unbiased search of the survey's 12 billion pixels.

In order to take advantage of the additional information contained in the images themselves, we have supplemented our catalog search with a direct source search at the positions of the 13,302 Hipparcos stars that fall within FIRST images. Our standard sourcefinding algorithm HAPPY (see WBHG for a complete description) was run on $1^{\prime}$ square boxes centered at each stellar location with the threshold set at 0.45 mJy (corresponding to a CLEAN-bias corrected flux density of $0.7 \mathrm{mJy}$ ). The algorithm search was truncated if it found more than five sources in a single box (indicative of a noisy image). Since the noise in VLA snapshot images is not strictly Gaussian, we determined the rate of false hits empirically by retaining all sources that matched a stellar position (always known to better than $0.1^{\prime \prime}$ ) to within $10^{\prime \prime}$.

Of the $>13,0001 \operatorname{arcmin}^{2}$ boxes searched, 47 yielded "detections" above the specified map threshold of $0.45 \mathrm{mJy}$ within $2^{\prime \prime}$ of the stellar position. Each of these was examined by eye in order to assess the local noise properties of the map, eliminate unCLEANed low-level sidelobes, and eliminate cases in which the detection represented a component of a larger, extended source. Twenty-two sources survived this screening, of which 13 are Hipparcos stars identified above. Of the nine new objects, four are previously known radio stars, lending considerable credence to our methodology. All four of these stars, plus three of the remaining five sources, are detected in the RASS-BSC; in addition, these seven stars are all brighter than $m_{v}=8.5$, while only $40 \%$ of all Hipparcos stars (but $100 \%$ of our cataloged detections) are this bright. We conclude that these seven are high-probability radio star detections and list their properties in Table 1.

The two objects missing from the RASS-BSC are both A stars. Both have offsets near the acceptable limit of $2^{\prime \prime}$ and are near the limit of detectability in their respective radio images. A false rate calculation based on the total number of unscreened candidates with offsets in the range $5^{\prime \prime}$ to $10^{\prime \prime}$ is consistent 
with both being chance coincidences. Without followup confirmation, these detections must be treated as provisional and we include them in Table $2 \mathrm{~A}$.

For stars in the Hipparcos catalog, then, the effective threshold of our survey for stellar radio sources is $0.7 \mathrm{mJy}$ when the CLEAN bias of $0.25 \mathrm{mJy}$ is added to the map flux densities. The survey is not, however, complete to this threshold, since over nearly half the survey area, the noise level is such that a $0.45 \mathrm{mJy}$ map flux density would not represent a $3 \sigma$ detection.

The large number of trials necessary to repeat this procedure for the Tycho catalog and, as important, the less accurate Tycho positions, renders such an exercise unproductive. The same comments apply to the GSC. For the 25pc catalog, we have effectively conducted this deeper survey by examining each image containing a catalog entry by eye.

\section{Accounting for stellar variability}

\subsection{Variability of the detected stars}

Stellar radio sources are almost all highly variable objects. For each star in Tables 1 and 2 as well as those in the GSC match list, we have derived a flux density or upper limit from each grid image in which it appeared (see Helfand et al. 1999 (in preparation) for the details of our source variability database and analysis procedures). Six of the 16 stars detected in at least one of the grid images show clear $(>4 \sigma)$ evidence of variability. Of these, 4 sources are also detected in the NVSS, and one additional variable is confirmed via that comparison. Maximum and minimum flux densities are recorded in the final column of Table 1, where we also include optical variability information from the Hipparcos catalog. Since only a tiny fraction of FIRST sources are variable $\left(<10^{-3}\right.$; Helfand et al. 1997), this relatively large representation of variable sources is yet further confirmation that the stellar identifications are correct. In contrast, none of the stellar candidates from the GSC match are variable, consistent with our conclusion that virtually all are false coincidences.

\subsection{Searching for additional sources}

A final search with the Hipparcos and Tycho catalogs takes advantage of the inherent variability of radio stars and the overlapping coverage of the FIRST survey design. Nearly every point in the survey area is observed more than once (from two to five times), with time differences ranging from 3 minutes to 3 years. Thus, a flaring source could conceivably appear above threshold in a single image, while falling below the threshold in the final map after several observations of the star in the quiescent state are averaged in.

Before producing the final FIRST coadded images, we run our source detection algorithm on each of the individual "grid" pointings, generating a catalog of over 1,425,000 sources, many of which are multiple entries. Matching the Hipparcos positions to this cata$\log$ yields four additional sources coincident to within $2^{\prime \prime}$ (all four have offsets $\leq 1^{\prime \prime}$ ). One of these also appears in the detailed image analysis for sources below the nominal survey threshold reported above $(\S 4)$ and so already appears in Table 1 . The second detection is of a $1.2 \mathrm{mJy}$ point source that is coincident with a $m_{v}=7.5 \mathrm{G} 2 \mathrm{~V}$ star. Since even for this larger cata$\log$, the chance coincidence rate is only $\sim 1.4$ for the entire Hipparcos catalog and $\sim 0.4$ for stars brighter than $m_{v}=7.5$, this match is likely to be real; as it is also detected as a bright RASS-BSC source, we include it in Table 1 . The third candidate, an $11 \mathrm{mJy}$ "source" is actually a sidelobe from a 3 Jy source $\sim 12^{\prime}$ away (such objects usually do not make it into the FIRST catalog because they are either eliminated in the weighting and coadding of the thirteen overlapping pointings which comprise a final image, or they are flagged explicitly as sidelobes by an algorithm we have designed expressly for that purpose). The fourth star has the largest offset $\left(1.03^{\prime \prime}\right)$, is the faintest $\left(m_{v}=9.8\right)$, and is of spectral type F8. These latter two candidates, neither of which are X-ray detected, most likely represent the expected $\sim 2$ chance coincidences, and we include them in Table $2 \mathrm{~B}$ where the other unlikely candidates are collected.

The Tycho catalog was also compared to the grid image catalog, and 36 matches were found; the expected false rate is 8 . Of the 36 matches, 14 are real radio stars reported above from the various matching strategies, three are known to be either Planetary Nebulae or galaxies, 6 are obvious sidelobes or noise in the raw grid images, and two are extragalactic double radio sources; the latter two categories account for the expected false rate. This leaves nine stars, of which at most a few are expected to be false. Three of the nine were reported above from the Tycho-FIRST catalog match (as were several of the demonstrably false matches); one new coincidence is an X-ray source, and we include it in Table 1. After screening out obvious 
sidelobes by visual inspection of the grid images, we record in Table $2 \mathrm{~A}$ the remaining matches which, as in the rest of that Table, may be one-third to one-half false.

\section{Discussion}

\subsection{Previously known radio stars}

Wendker 1995 has compiled a catalog of all stellar radio observations reported in the literature which is complete through June 30, 1993 and contains considerable information for a year beyond that date. It includes over 3000 distinct stars of which 821 have been detected at least once in the meter-wave to submillimeter-wave band, providing a rather complete census of the stellar radio work previously carried out in our survey region. The positions of all such stars included in this region were examined. In addition, we performed our own literature search on all objects detected from the above catalogs to cover the period 1993.5 to the present.

The Wendker catalog contains 146 entries within the FIRST survey region. Of these 125 are reported only as upper limits which, apart from a handful of cases, are not as constraining as the FIRST results ${ }^{8}$. The other 21 objects have all been seen at least once in the 0.3-30 GHz band. We have detected nine of these objects, and we record the range of flux densities cited in the literature in column 18 of Table 1 (including one additional star detected subsequent to the completion of Wendker's catalog). Of the remaining dozen objects, almost all have shown evidence for variability down to limits well below $0.7 \mathrm{mJy}$ at $20 \mathrm{~cm}$, so our nondetection is unsurprising. Indeed, only seven of the stars undetected in FIRST have ever been reported at flux densities above $0.7 \mathrm{mJy}$ at $20 \mathrm{~cm}$ (or sufficiently above this limit at a somewhat higher frequency as to make it likely that we would have detected the star if it were at the previously reported level of activity). We list the 12 radio stars which we did not detect in Table 3 along with their historical range of flux densities at the lowest frequency available and a three-sigma upper limit from the relevant FIRST image. Nine of the twelve have been previously detected as X-ray sources (although only seven appear in the RASS-BSC), both confirm-

\footnotetext{
${ }^{8}$ We include stars in Wendker's compilation which have only been detected at frequencies above $30 \mathrm{GHz}$ as upper limits in this discussion of centimetric radio emission.
}

ing the general correlation of X-ray and radio activity and admitting the possibility that the handful of our radio star candidates without X-ray counterparts are real detections.

While this heterogeneous, optically selected sample may include a different mean level of variability than our unbiased, radio-selected sample, our failure to detect 7 of the 17 previously known stars which have been seen above our threshold suggests an incompleteness in our radio-selected sample of roughly a factor of two.

\subsection{The types of radio stars detected}

While we have not yet carried out optical followup observations of the stars detected here for the first time at radio wavelengths, some of the detections are not unexpected. Two of the new radio emitters are RSCVn binaries and another is a BY Draconis system; these two classes are among the most luminous of stellar radio emitters. Three others are dMe stars which have also long been known as radio sources. The remaining new detections are F, G, and K stars, most without luminosity classes; three are cataloged optical variable stars. V340 Gem is extremely luminous (up to $L_{r} \sim 10^{18} \mathrm{ergs}^{-1} \mathrm{~Hz}^{-1}$ at its brightest), while the star with the brightest mean radio flux, FIRST J142104.8+385914, has an anomalously high X-ray-to-radio flux ratio. These objects may be among the first of the new classes of radio emitters that an unbiased survey such as the one we have conducted here can be expected to detect.

\subsection{The fraction of radio-bright stars}

Even at a threshold of $\sim 0.7 \mathrm{mJy}$, stellar radio emission remains rare. In Figure 1, we plot the fraction of stars detected as a function of $V$-magnitude. The data for $V<10$ include only detections and nondetections from the Hipparcos complete (magnitudelimited) sample in order to avoid biasing the estimate high as a consequence of the inclusion of fainter radio stars that were added to the Hipparcos input catalog because they were radio stars. The $10<V<11$ bin is derived from the Tycho matches corrected for chance coincidences (and thus applies to the slightly higher flux density threshold of $1.0 \mathrm{mJy}$ ). The error bars represent the Poisson errors based on the number of detected stars in each bin. The line representing the fraction of detected stars as a function of magnitude, $\log f(m)=-0.3 m$, is plotted for comparison and has 


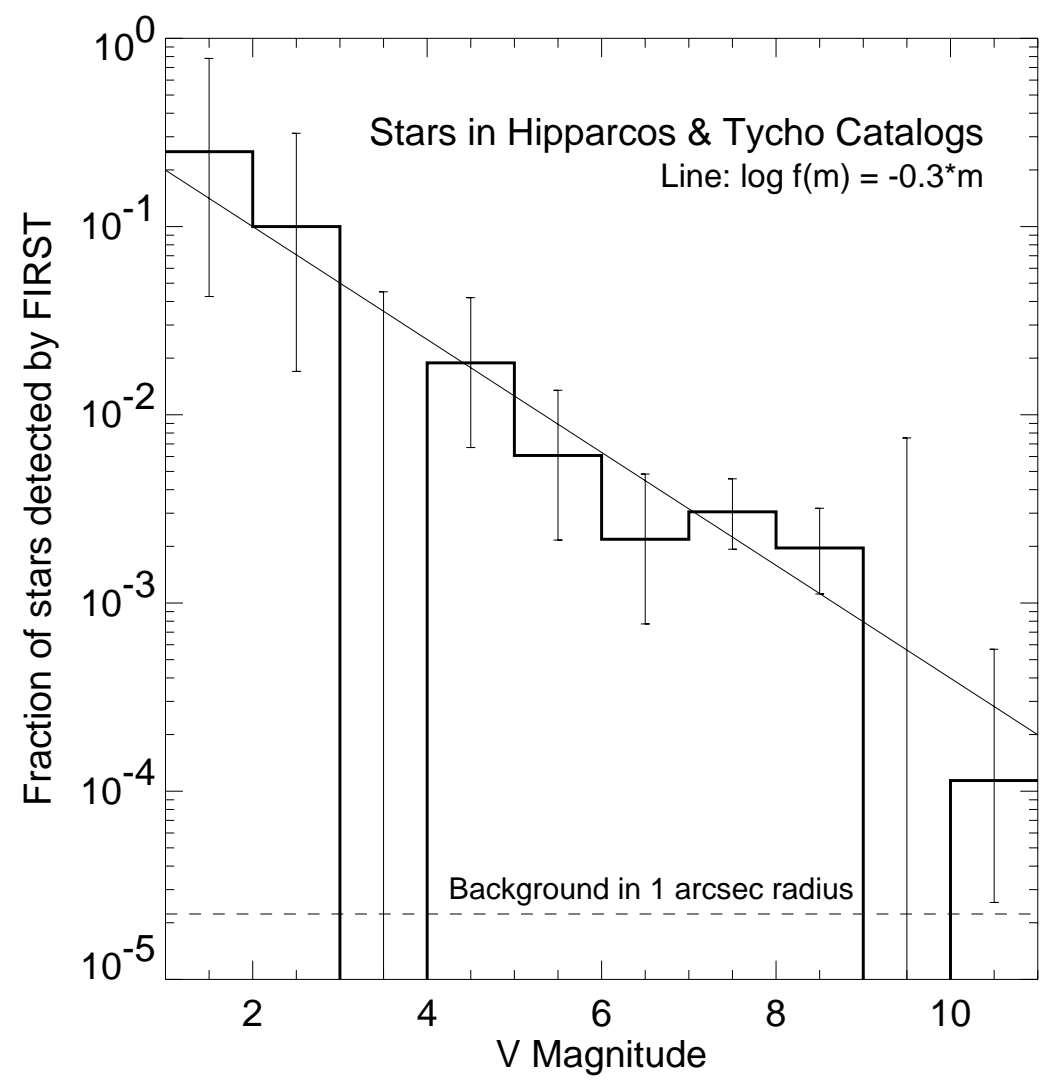

Fig. 1. - The fraction of radio detections as a function of stellar magnitude. For the magnitude range $1<V<10$, we use the complete subsample of the Hipparcos catalog to calculate the detected fraction; for $10<V<11$ we use the statistics from our Tycho catalog match. As a consequence, the radio detection limits are slightly different (see text). The solid line is simply an empirical representation of the trend and has no physical meaning. The dashed horizontal line represents the chance coincidence rate for an error circle of radius $1^{\prime \prime}$. 
no physical significance.

The fraction of stars detected falls below $1 \%$ at the threshold for naked-eye stars, and reaches $10^{-3.5}$ at $\mathrm{V}=10.5$. Owing to the highly variable nature of stellar radio emission and the incompleteness of our survey below $1 \mathrm{mJy}$ noted above, this curve represents a lower limit; however, given the fraction of previously known radio stars we detect, it is probably not low by more than a factor of $\sim 2$. The chance coincidence rate for radio source positions accurate to $1.0^{\prime \prime}$ is indicated; note that the empirical curve for the fraction of detectable stars intercepts this limit at $V \sim 14.5$, while the corresponding limit for $2^{\prime \prime}$ positional uncertainties adopted in this paper is at $V \sim 12.5$.

As Figure 1 suggests, our study has not exhausted the number of stellar radio sources which will ultimately be identified in the FIRST survey. To estimate the number of additional stars we could detect if good proper motions were available, we have used the Bahcall-Soniera (Bahcall and Soneira 1980) star count models for the FIRST survey area scaled up by a factor of two to match the observed GSC star counts in the range $5<V<12$. Multiplying the detectedfraction line in Figure 1 by this magnitude distribution yields predicted stellar detections of 44 objects with $V<11$ and 63 objects with $V<12.5$ (above which the GSC is complete and our $2^{\prime \prime}$ matching criterion is applicable), once adequate proper motion measurements from GSC-II become available. For specific classes of stars (e.g., dMe stars that could be selected by their very red colors or stellar X-ray detections), we can substantially reduce the false rate and therefore go considerably fainter as indicated by our detection of two stars with $V>14$ in this paper; at $V<15$, we expect $\sim 100$ radio star detections in the current FIRST region. Thus, combining a multicolor photometric survey such as the Sloan Digital Sky Survey with proper motions from GSC-II could easily quadruple the number of objects detected.

\subsection{Summary and conclusions}

We have completed the FIRST unbiased search for stellar radio emission by combining our $20 \mathrm{~cm}$ VLA radio survey with several astrometric catalogs of bright stars. We detect 10 of the 17 stars in this $4760 \mathrm{deg}^{2}$ region of sky ever seen above $0.5 \mathrm{mJy}$ at $20 \mathrm{~cm}$, and add a total of 16 new high-confidence detections, doubling the number of known stellar radio emitters; seven additional candidates for which the evidence is weaker are also identified. Although this technique permits us to discover new classes of radio stars and to extend the range of properties of known classes of emitters, radio stars remain rare: $<10^{-4}$ of FIRST radio sources are identified here as stars, and we detect only $3 \times 10^{-4}$ of the stars in the Tycho catalog. The GSC-II catalog offers the promise of substantially enhancing the number of stellar sources identified by the FIRST survey, with several dozen additional detections in the current survey area.

The FIRST survey project has been supported by grants from the National Geographic Society, the National Science Foundation (AST-94-19906), NASA (NAG5-6035), NATO, IGPP, Columbia University, and Sun Microsystems. Part of this work has been performed under the auspices of the U.S. Department of Energy by LLNL under contract W-7405-ENG-48. We are grateful for significant contributions to this effort by Don Neill. DJH thanks the Institute for Astronomy, University of Cambridge and the Raymond and Beverly Sackler Visiting Lecturer Fund for hospitality and support during the completion of this work. This research has made use of data obtained from the High Energy Astrophysics Science Archive Research Center (HEASARC) provided by NASA's Goddard Space Flight Center. This is Columbia Astrophysics Laboratory Contribution No. 673.

\section{REFERENCES}

Abbott, D.C., Bieging, J.H., Churchwell, E. and Torres, A.V. 1986 ApJ, 303, 239

Bahcall, J.N. and Soneira, R.M. 1980, ApJS, 44, 73

Becker, R.H., White, R.L., and Helfand, D.J. 1995, ApJ, 450, 559

Becker, R.H., White, R.L., Helfand, D.J., Gregg, M.D., and McMahon, R. G. 1996 in Radio Emission from the Stars and the Sun, eds. J.M. Paredes and R. Taylor, ASP Conf. Ser. 93, 422

Braes, L.L.E. and Miley, G.K. 1971, Nature, 232, 246

Chanmugam, G. and Dulk, G.K. 1982, ApJ,255, L107

Cohen, M. Bieging, J.H., and Schwartz, P.R. 1982, ApJ, 253, 707

Condon, J.J., Cotton, W.D., Greisen, E.W., Yin, Q.F., Perley, R.A., Taylor, G.B., and Broderick, J.J. 1998, AJ, 115, 1693 
Condon, J.J., Kaplan, D.L., and Yin, Q.F. 1997, BAAS, 191, 1402

Davis, R.J., Lovell, B., Palmer, H.P., and Spencer, R.E. 1978 Nature, 273, 647

Drake, S.A., Abbott, D.C., Bastian, T.S., Bieging, J.H., Churchwell, E. Dulk, G. and Linsky, J.L. 1987, ApJ, 322, 902

Gary, D.E. and Linsky, J.L. 1981, ApJ 250, 284

Gibson, D.M. and Hjellming, R.M. 1974 PASP, 86, 652

Gliese, W. and Jahreiss, H. 1991, Preliminary Version of the Third Catalog of Nearby Stars (Heidelberg: Astron. Rechen-Institut)

Gudel, M. and Benz, A.O. 1993, ApJ, 405, L63

Helfand, D.J., Das, S.R., Becker, R.H., White, R.L., and McMahon, R.G. 1997, in Blazar Continuum Variability, ed: H. Miller, J. Webb and J. Noble, ASP Conf. Series, Vol. 110, 214

Hey, J.S. 1973 in The Evolution of Radio Astronomy (London:Elek Science), p14

Hjellming, R.M. 1988 in Galactic and Extragalactic Radio Astronomy, ed. G.L. Verschuur and K.I. Kellerman (Berlin: Springer-Verlag), p381

Hjellming, R.M. and Wade, C.M. 1970 ApJ 162, L1

Hjellming, R.M. and Blankenship, L.C. 1973, Nature Phys. Sci., 243, 81

Hjellming, R.M. and Wade, C.M. 1971, ApJ, 164, L1

Høg, E. et al. 1997, A\&A, 323, L57

Lasker, B.M., Sturch, C.R., McLean, B.J., Russell, J.L., Jenkner, H. and Shara, M.M. 1990, AJ, 99, 2019

Lim, J. and White, S.M. 1995, ApJ, 453, 207

Lovell, B. 1964, The Observatory, 84, 191

McMahon, R.G., Helfand, D.J., White, R.L. and Becker, R.H. 1998, (in preparation)

Padin, S., Davies, R.J. and Bode, M.F. 1985, Nature, 315,306

Perryman, M.A.C. 1997, A\&A, 323, L49
Röser, S. and Bastian, U. 1991 'The PPM Star Catalog' (Heidelberg:Spektrum Akademisher Verlag)

Ryle, M., Smith, F.G., and Elsmore, B. 1950, MNRAS 110, 508

Spangler, S.R., Shawhan, S.D., and Rankin, J.M. 1974, ApJ, 190, L129

Turner, K. 1985 in Radio Stars, eds. R.M. Hjellming and D.M. Gibson (Dordrecht: Reidel), p283

Voges, W. et al. 1996, IAUC 6420

Wade, C.M. and Hjellming, R.M. 1972, Nature, 235, 270

Wendker, . 1995. A\&AS, 109, 177

White, R.L. and Becker, R.H. 1982, ApJ, 262, 657

White, R.L., Becker, R.H., Helfand, D.J., and Gregg, M.D. 1997, ApJ 475, 479

This 2-column preprint was prepared with the AAS IATEX macros v4.0. 